\title{
Plant nanocellulose and its composite hydrogel membranes-based polyvinyl alcohol/hyaluronic acid for biomedical applications: Extraction, characterization, and in vitro bioevaluation
}

\author{
Yasmein Hussein ${ }^{1}$, Elbadawy A. Kamoun ${ }^{1,2^{*}}$, Samah A. Loutfy ${ }^{1,3}$, Rehab Amen ${ }^{4}$, Tarek H. Taha ${ }^{5}$, Ahmed S. Mansour ${ }^{6}$, \\ Ahmed I. Abdel-Salam ${ }^{7}$ Mahmoud Amer ${ }^{8}$ \\ ${ }^{1}$ Nanotechnology Research Center NTRC, The British University in Egypt BUE, El-Sherouk City, Egypt. \\ ${ }^{2}$ Polymeric Materials Research Department, Advanced Technology and New Materials Research Institute ATNMRI, City of Scientific Research and \\ Technological Applications SRTA-City, New Borg Al-Arab City, Egypt. \\ ${ }^{3}$ Virology and Immunology Unit, Cancer Biology Department, National Cancer Institute, Cairo University, Giza, Egypt. \\ ${ }^{4}$ National Institute of Laser Enhanced Science NILES, Cairo University, Giza, Egypt. \\ ${ }^{5}$ Environmental Biotechnology Department, Genetic Engineering and Biotechnology Research Institute, City of Scientific Research and Technological \\ Applications SRTA-City, New Borg Al-Arab City, Egypt. \\ ${ }^{6}$ National Institute of Laser Enhanced Science NILES, Cairo University, Giza, Egypt \\ ${ }^{7}$ Nanotechnology Research Center NTRC, The British University in Egypt BUE, El-Sherouk City, Egypt. \\ ${ }^{8}$ Zoology Department, Faculty of Science, Cairo University, Cairo, Egypt
}

\section{ARTICLE INFO \\ Received on: 02/10/2020 \\ Accepted on: 19/12/2020 \\ Available online: 05/01/2021}

\section{Key words:}

Cellulose nanocrystals, extraction, PVA/hyaluronic acid, composite hydrogel membranes, bioevaluation tests.

\begin{abstract}
Needle-like cellulose nanocrystals (CNCs) were prepared from useless rice straw, which posed problems of environmental pollution in Egypt during its disposal in the burning process. Cellulose was obtained using an alkaline treatment, followed by a bleaching process and acid hydrolysis producing a high yield of CNCs $\sim 12 \pm 3$ $(\%, w / w)$. Resultant CNCs were instrumentally characterized by FTIR, XRD, transmission electron microscopy (TEM), Brunauer-Emmett-Teller, and particle potential analyses; moreover, antimicrobial activity, hemolysis, and cytotoxicity were bioassessed. XRD exhibited an elevated crystallinity index of approximately $98 \%$, and thermal decomposition of thermogravimetric analysis thermograph ranged from $\sim 245$ to $480^{\circ} \mathrm{C}$. The TEM investigation showed that the nonaggregated needle-like nanocrystals diameter ranged from $\sim 20$ to $60 \mathrm{~nm}$. Interestingly, CNCs at high concentrations $(1,000 \mu \mathrm{g} / \mathrm{ml})$ did not affect the viability of HFB-4 and WI38 human normal cell lines. Polyvinyl alcohol (PVA)/hyaluronic acid (HA) hydrogel membranes were reinforced with various concentrations of CNCs $[0.25,0.5$, and $1.0 \%(w / v)]$ and prepared by the solution-casting method using citric acid as a crosslinker. The results revealed that using $\mathrm{CNCs}$ as nanofillers up to $0.5 \%$ enhanced dramatically the mechanical properties of membranes and exhibited good swelling capacity, biocompatibility, and antimicrobial activity, compared to hydrogel free CNCs. Therefore, the PVA/HA/CNCs membranes might have great potential in wound dressing application and it can be regarded as suitable to be developed into targeted nanocarriers or nanofillers for biomedical applications.
\end{abstract}

\section{INTRODUCTION}

Cellulosic materials attract much attention of many researchers as reviewable, low cost, and available sources

\footnotetext{
${ }^{*}$ Corresponding Author Elbadawy A. Kamoun, Nanotechnology Research Center NTRC, The British University in Egypt BUE, El-Sherouk City, Egypt;Polymeric Materials Research Department, Advanced Technology and New Materials Research Institute (ATNMRI), City of Scientific Research and Technological Applications (SRTA-City), New Borg Al-Arab City, Egypt. E-mail:badawykamoun@yahoo.com
}

for applying for several purposes, such as biomedical and pharmaceutical applications. Furthermore, these researches are extended to transform cellulose materials into nanoscale to meet the demand of various and specific applications. Nanocellulose is the main affluent renewable and environmental friendly polysaccharide biopolymer in nature. Cellulose consists of a long chain of polysaccharides $D$-glucose linked via $\beta$-1,4 glycoside bond (Mu et al., 2019). According to crystallinity, size, and mechanical tensile, nanocellulose is divided into three types: cellulose nanocrystals or nanowhiskers (CNCs) or cellulose whiskers, cellulose nanofibrils, and microfibrillated cellulose (Sharma et al., 2019). Recently, CNCs are the major nanocellulose 
type that has occupied the interest of many researchers owing to their biophysicochemical characteristics, for example, nanoscale dimensions (typically $\sim 2-50 \mathrm{~nm}$ in $\mathrm{W} \times 100-2,000 \mathrm{~nm}$ in 1 ) (El Miri et al., 2015), high $s p$. surface area $\left(>100 \mathrm{~m}^{2} / \mathrm{g}\right)$, low density $\left(1.6 \mathrm{~g} / \mathrm{cm}^{3}\right)$ (Du et al., 2019), high crystallization (Yin et al., 2019), thermal stability, hydrophilic absorbent, and easy modification because of a huge surface containing $-\mathrm{OH}$ and sulfate ester groups that are incorporated during sulfuric acid hydrolysis (Bespalova et al., 2017). Moreover, CNCs have outstanding mechanical properties enabling them to serve as an ideal reinforcement nanofiller, whereas their Young's modulus is about 100-200 GPa (Pelegrini et al., 2019). Thus, CNCs can serve as ideal reinforcement nanofillers and can incorporate into different natural or synthetic polymers matrices surfaces.

To date, there are many sources for isolation of CNCs which were explored, including lignocellulosic biomass, bacteria, plants, and animals (Sharma et al., 2019). Currently, CNCs could be isolated by efficient methods, for example, acid hydrolysis, enzymatic hydrolysis, and mechanical treatment processes (Sharma et al., 2019). However, sulfuric acid hydrolysis represents the most famous route for preparing CNCs where a concentrated solution of sulfuric acids involves hydrolysis and deducting of amorphous parts of cellulose, while the crystalline parts remain intact (Sharma et al., 2019). Nowadays, CNCs have emerged in various medical applications such as bone and cartilage regeneration, dental implants, drug delivery, and wound healing (Halib et al., 2017) due to their biodegradability (El Miri et al., 2015) and biocompatibility properties. In addition, CNCs did not affect the viability of a wide range of human cell lines at a concentration of $250 \mu \mathrm{g} / \mathrm{ml}$.

As previously reported, the synthesis of outstanding mechanical performance scaffold composed of CNCs reinforced chitosan/pectin for using as biomaterials for tissue engineering and bone regeneration (Ghorbani et al., 2020). Lastly, (Singla et al., 2017a, b) reported the ability of CNCs to enhance healing of chronic and diabetic wounds ( $99 \%$ wound closure) via reducing inflammation, enhancing collagen deposition, and increasing the proliferation rate of epithelial and fibroblast cells. These properties widen the use of CNCs as nanofillers into biocomposites for wound dressing application (Yin et al., 2019). In fact, bacterial nanocellulose seems to be preferable for medical applications since it was biosynthesized as a natural extracellular product with high purity and without any need for chemical treatment (Halib et al., 2017). However, CNCs have some drawbacks that might restrict their scaling up during clinical applications, like sophisticated isolation with the high cost and low yield. For these reasons, numerous efforts have been made to isolate CNCs from low cost, renewable agriculture residues, such as rice straw and sugarcane bagasse (Khattab et al., 2020). Rice straw was found as one of the renewable sources of CNCs containing about $38.3 \%$ cellulose. Updated researches have shed light on the utilization of CNCs from rice straw as reinforcing fillers in biocomposite scaffolds to improve mechanical properties of wound dressings.

Hyaluronic acid (HA) is a natural polymer composed of $\beta$-(1,4)-linked $D$-glucuronic acid and $\beta$-(1,3) $N$-acetyl- $D$ glucosamine units. HA is one of the essential components in ECM that is involved in wound dressing materials owing to its ability in reducing inflammation, enhancing proliferation, and migration of skin cells, inducing angiogenesis and collagen deposition (Hadisi et al., 2020). Therefore, HA was demonstrated as a promising candidate substance for wound dressing materials. However, the lack of its mechanical properties that are required for the growth of living cells may hinder its application; therefore, there is a need to incorporate other reinforcing agents to overcome this limitation.

This encouraged us to improve the durability and stability of chemically crosslinked polyvinyl alcohol (PVA)/HA hydrogel membranes using low cost and renewable nanofillers, for example, CNCs that are extracted from rice straw pulp. Moreover, this approach represents a suitable economic and scientific method to manage the environmental problem of rice waste named "Black Cloud" released from the burning of rice straw in some countries like Egypt, India, China, and Thailand (El-Dewany et al., 2018) and also turns this environmental hazard into a renewable source of CNCs for large-scale industries. In addition, the current study aims to evaluate the effect of CNCs' incorporation on mechanical properties of hydrogel membranes and bioevaluations, such as antimicrobial activity, hemocompatibility, and cell viability of normal human skin melanocyte and lung fibroblast cells.

\section{MATERIALS AND METHODS}

\section{Materials}

CNCs were isolated from rice straw (Kafr El-Sheikh rice farms, Egypt). Sodium hydroxide (97\%) was purchased from Loba Chemie, India. Sodium chlorite $\left(\mathrm{NaClO}_{2}, 80 \%\right)$ was obtained from Fluka, Germany. Acetic acid glacial (99.0\%) was obtained from Merkmillipore, UK. Sulfuric acid (purity 95\%-98\%) and citric acid (CA) anhydrous were obtained from Sigma-Aldrich Chemie GmbH, Steinheim, Germany. PVA $\left(M_{\mathrm{wt}} \sim 72,000 \mathrm{~g} / \mathrm{mol}\right.$, $86 \%$ hydrolyzed) was obtained from Loba Chemie, India. HA $\left(M_{\mathrm{wt}}\right.$ $\sim 600-750 \mathrm{kDa}$ ) was purchased from Shanghai Jiaoyuan Industry Co., Ltd., China.

\section{Preparation of CNCs and PVA/HA/CNCs hydrogel membranes}

\section{Synthesis of CNCs from rice straw}

CNCs were extracted from rice straw according to the previously published procedure of $\mathrm{Lu}$ and Hsieh (2012), with a slight modification, including dewaxing and alkaline treatment, followed by bleaching or delignification. It was exchanged in a sequence of chemical processes with alkaline treatment using a high concentration of $\mathrm{NaOH}$ instead of $\mathrm{KOH}$ at a pressure rotary reactor, followed by a delignification process. The extraction of CNCs from rice straw was carried out into two stages: isolation of cellulose fibers from rice straw, followed by transformation of the isolated cellulose into CNCs. In brief, rice straw was washed and grained and then dewaxed by $2: 1(\mathrm{v} / \mathrm{v})$ mixture solution of toluene/ ethanol for 20 hours. The dewaxed rice straw was alkaline treated with $10 \%(w / v) \mathrm{NaOH}$ and the mixture was boiled for 60 minutes at rotator pressure reactor; then the blend was kept overnight at ambient conditions. The residues were rinsed with distilled water till reaching neutral $\mathrm{pH} \sim 7.0$. The delignification was conducted by adding $1.0 \%(w / v) \mathrm{NaClO}_{2}$ and the mixture was boiled for a further 120 minutes at $\mathrm{pH} \sim 4.0$ using glacial acetic acid. Then, the solid residue was washed several times till reaching $\mathrm{pH} \sim 7$, again. The delignification process was repeated with three technical replicates to dissolve the lignin and obtain cellulose with yellowish 
white color. The extracted cellulose fibers were transformed into CNCs by sulfuric acid hydrolysis process according to protocol carried out by Lu and Hsieh (2012). Cellulose fibers were mixed with $64 \%(w / w)$ of sulfuric acid with continuous stirring for 1 hour at ambient conditions. The extracted CNCs suspension was washed several times to remove the excess of acid and reaching neutral $\mathrm{pH} \sim 7.0$. The resultant solution was centrifuged at 15,000 rpm for 30 minutes; then, CNCs were dried using lyophilizer for 48 hours for further use.

The extraction was obtained as $35 \%$ cellulose, $20 \%$ hemicellulose, $5 \%$ lignin, and 14\% silica. Then, the cellulose was transformed into CNCs by acid hydrolysis reaction with yield range of $\sim 35-45 \%(w / w)$, with respect to extracted cellulose, and $12 \% \pm 3 \%(w / w)$ with respect to rice straw.

\section{Synthesis of PVA/HA/CNCs composite hydrogel membranes}

PVA/HA and PVA/HA/CNCs hydrogel membranes were prepared by the solution-casting method as published elsewhere (Xu et al., 2013). First, PVA solution (10\%,w/v) and HA solution $(2 \%, w / v)$ were prepared and dissolved separately in distilled water at room temperature. Optimization of PVA/HA polymer blend membrane was formed through mixing PVA and HA together at various volume ratios of 80:20,60:40, and 50:50, respectively, for 3 hours at $50^{\circ} \mathrm{C}$ in a closed vial to avoid bubbles formation and for ensuring full homogeneity. One $\mathrm{ml}$ of glycerol was added as a plasticizer for each membrane sample. CA was added to PVA/HA mixture as crosslinker with varying concentrations $(2.5 \%, 5 \%$, and $10 \%, w / v)$ with stirring for 1 hour at room temperature.

Series of PVA/HA/CNCs membranes were prepared by dispersion of CNCs at different weights $(0 \%, 0.25 \%, 0.5 \%$, and $1.0 \%, w / v)$ as nanofillers into $10 \%(w / v)$ of CA crosslinked PVA/ HA (80:20) solution mixture. Solutions were stirred at $50^{\circ} \mathrm{C}$ for 2 hours to obtain homogenous well-dispersed PVA/HA/CNCs blend mixture, followed by adding $1 \mathrm{~mL}$ of glycerol for each membrane sample. PVA/HA and PVA/HA/CNCs solutions were ultrasonicated for further 1 hour to ensure removal of air bubbles, and then resultant homogeneous solutions were poured into a glass Petri dish, followed by thermal treating in an oven at $80^{\circ} \mathrm{C}$ for 18 hours for initiating the crosslinking reaction.

\section{Instrumental characterization of CNCs}

\section{FTIR}

The chemical structure of CNCs was determined by FTIR (Bruker Vertex 70, Germany). IR fingerprints were recorded between 4,000 and $400 \mathrm{~cm}^{-1}$ using transmittance modes.

\section{SEM}

The surface morphology of membranes was investigated by SEM (ESEM, Quattro S, Thermo Scientific) at an accelerating voltage of $5 \mathrm{Kv}$ and original magnification at 4,000x and 10,000x.

$X R D$

The crystallinity of CNCs was verified by XRD using XRD Panalytical Empyrean, England. Radial scans of intensity were carried out at room conditions $\left(2 \theta=5^{\circ}-80^{\circ}\right)$ with a step increment of $0.02^{\circ}$ per second. The crystallinity index (CrI) was calculated using the following empirical equation (El Miri et al., 2015; Pelegrini et al., 2019; Segal et al., 1959):

$$
\operatorname{CrI}(\%)=\left(\mathrm{I}_{002}-\mathrm{I}_{\mathrm{am}} / \mathrm{I}_{002}\right) \times 100,
$$

where $\mathrm{I}_{002}$ is the maximum intensity of diffraction and $\mathrm{I}_{\mathrm{am}}$ is the amorphous part of the sample, located at minimum intensity.

\section{Zeta measurements}

Zeta sizer model (Malvern Instruments, UK) was utilized for determining the zeta potential value of CNCs nanoparticles.

\section{Brunauer-Emmet-Teller (BET)}

The nitrogen adsorption-desorption isotherms were degassed at $35^{\circ} \mathrm{C}$ under vacuum for 24 hours, which was carried out to determine the specific surface areas of CNCs; samples were analyzed at $77 \mathrm{~K}$ by a surface area and porosity analyzer model (Quantachrome 11.04, Anton Paar, Ashland, VA).

\section{Transmission electron microscopy (TEM)}

Particle size/shape and size distribution profile of CNCs were measured by TEM model (JEOL JEM-2100, JEOL, Akishima, Japan). A drop of dispersed sample was deposited onto a carbon-coated copper grid and left to evaporate at room temperature. CNCs' size distribution was determined using Image $\mathrm{J}$ analysis software.

\section{Mechanical properties}

The tensile strength of membranes was operated by standard uniaxial tensile test (Z050, Zwick Roell AG, Ulm, Germany).

\section{Thermogravimetric analysis (TGA)}

The thermal degradation of CNCs was measured by the TGA model (TGA-50, Shimadzu, Japan). The sample was operated under $\mathrm{N}_{2}$ gas with a heating rate of $10^{\circ} \mathrm{C} /$ minutes at $25-500^{\circ} \mathrm{C}$.

\section{Physicochemical characterization}

Swelling ratio (\%) of PVA/HA/CNCs composite hydrogel membranes

The swelling ratio of tested membranes was tested in distilled water at $37^{\circ} \mathrm{C}$. Composite hydrogel membranes were weighed individually $\left(\mathrm{W}_{\mathrm{e}}\right)$ and immersed separately in a Petri dish containing distilled water, $\mathrm{pH}$ 7. After specific time intervals, samples were reweighted after drying the excess water $\left(\mathrm{W}_{\mathrm{s}}\right)$. Then, swelling (\%) or water uptake of membranes was calculated with the following equation (Dehkordi et al., 2019):

$$
\text { Swelling }(\%)=\left(\mathrm{W}_{\mathrm{s}}-\mathrm{W}_{\mathrm{e}} / \mathrm{W}_{\mathrm{e}}\right) \times 100
$$

where $\mathrm{W}_{\mathrm{s}}$ and $\mathrm{W}_{\mathrm{e}}$ are the weight of the swollen and dried sample after soaking, respectively.

\section{In vitro hydrolytic degradation of $\mathrm{PVA} / \mathrm{HA} / \mathrm{CNCs}$ composite hydrogel membranes}

The hydrolytic degradation of membranes was estimated by immersing dried composite membranes with specific weight and dimension $(4 \times 4 \mathrm{~cm})$ in phosphate buffer saline (PBS) 
solution. The tested membranes were weighted again at time intervals after the removal of surfaced water and gently drying at ambient temperature (Hadisi et al., 2020). Then, the hydrolytic degradation was estimated using the following equation:

$$
\text { Weight loss }(\%)=\left[\left(\mathrm{W}_{0}-\mathrm{W}_{\mathrm{t}}\right) / \mathrm{W}_{0}\right] \times 100 \text {, }
$$

where $\mathrm{W}_{0}$ is the original weight of membrane and $\mathrm{W}_{\mathrm{t}}$ is the removed membrane from PBS and weighed at the function of specific incubation intervals.

\section{In vitro bioevaluation assays}

The biological behavior of the extracted CNCs, crosslinked $\mathrm{PVA} / \mathrm{HA}$, and $\mathrm{PVA} / \mathrm{HA} / \mathrm{CNCs}$ composite hydrogel membranes with $0.5 \%$ of CNCs' content was evaluated in terms of cell viability (\%), hemolysis affinity (\%), and antimicrobial activity.

\section{Cell culture and 3-(4,5-dimethylthiazol-2-yl)-2,5-diphenyltetrazolium bromide (MTT) assay}

Human normal lung fibroblast WI38 and human normal skin melanocyte HFB-4 cell lines were obtained from the American Tissue Culture Collection (ATCC) and supplied by VACSERA, Cairo, Egypt. Cells were kept in Dulbecco's Modified Eagle Medium (Gibco, South America) supplemented with 10\% heat-inactivated fetal bovine serum (Gibco, South America), 2\% penicillin-streptomycin (Lonza, NJ), 1\% sodium pyruvate (Lonza, NJ), and 1\% L-glutamine (Lonza, NJ) at standard conditions $\left(37^{\circ} \mathrm{C}\right.$ and $\left.5 \% \mathrm{CO}_{2}\right)$. The cells were kept in monolayer culture at $37^{\circ} \mathrm{C}$ under a humidified atmosphere of $5 \% \mathrm{CO}_{2}$. Cells were subcultured by trypsinization (170,000 U/1 trypsin and $200 \mathrm{mg} / 1$ EDTA; Lonza, NJ) and were kept in tissue culture laboratory at the Nanotechnology Research Center, British University in Egypt.

Cell viability (\%) and $\mathrm{IC}_{50}$ values of $\mathrm{CNCs}$ were evaluated using colorimetric MTT assay on HFB-4 and WI38 human cell lines at serial dilution ranged from 15 to $1,000 \mu \mathrm{g} /$ $\mathrm{ml}$ (Mosmann, 1983), at serial dilutions of $0,0.25,0.5,1,1.5$, and $2 \mathrm{mg} / \mathrm{ml}$ for both crosslinked PVA/HA and PVA/HA/CNCs composite hydrogel membranes (Mosmann, 1983).

The relative cell viability (\%) was calculated using the following equation:

The relative cell viability $(\%)=\left[\mathrm{OD}_{\mathrm{S}} / \mathrm{OD}_{\mathrm{C}}\right] \times 100$, Eq. (3)

where $\mathrm{OD}_{\mathrm{S}}$ is the mean optical density of the sample and $\mathrm{OD}_{\mathrm{C}}$ is the mean optical density of control. Obtained results were displayed graphically as cell viability (\%) against concentrations of tested materials using Graphpad Prism 7 software.

\section{Hemolysis test}

The biocompatibility of CNCs, PVA/HA, and PVA/HA/ $\mathrm{CNCs}$ membranes was investigated using hemolysis assay and the hemolysis (\%) was calculated using the following formula:

Hemolysis $\%=(\mathrm{TS}-\mathrm{NC}) /(\mathrm{PC}-\mathrm{NC}) \times 100$

where TS, NC, and PC are determined by absorbance values of the test sample, negative control, and positive control at $545 \mathrm{~nm}$, respectively (Raut et al., 2019).

\section{Antimicrobial activity}

The antimicrobial activity of CNCs, PVA/HA, and PVA/ $\mathrm{HA} / \mathrm{CNCs}$ against five pathogenic bacteria and yeast (Vibrio cholerae ATCC700, Candida albicans ATCC 700, Pseudomonas aeruginosa ATCC9027, Escherichia coli NCTC10418, and Klebsiella pneumonia ATCC13883) was investigated by disk diffusion method, as previously described (Fahmy et al., 2015).

\section{RESULTS AND DISCUSSION}

\section{Extraction and synthesis of CNCs from rice straw}

Figure 1a shows the steps of chemo-mechanical treatment for extracting CNCs. The first two steps (i.e., alkaline treatment and delignification) were carried out to break hydrogen bonds that connect cellulose with other components of rice straw resulting in the removal of noncellulosic constituents of rice straw, such as hemicellulose, lignin, and silica, as explained earlier. Subsequently, pure cellulose was transformed into CNCs by sulfuric acid hydrolysis treatment process as discussed elsewhere (Lu and Hsieh, 2012).

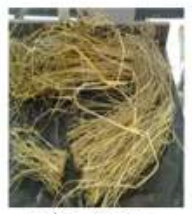

Rice straw

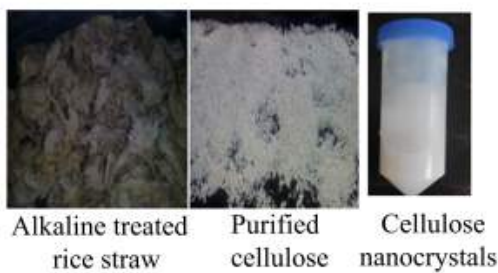

b

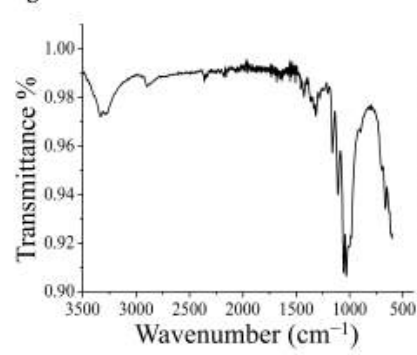

d
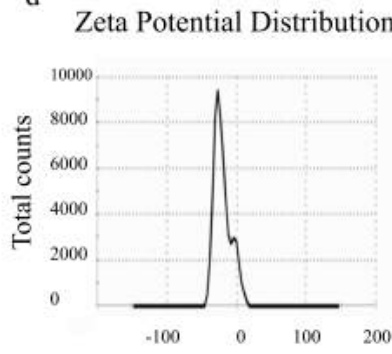

Apparent Zeta Potential $(\mathrm{mV})$

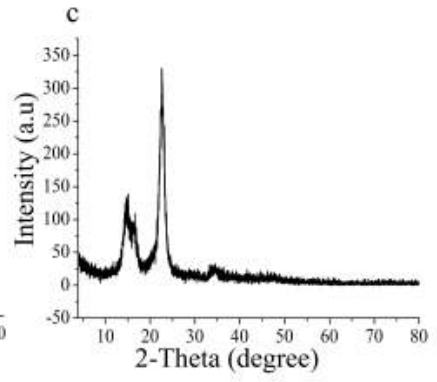

Size Distribution by number

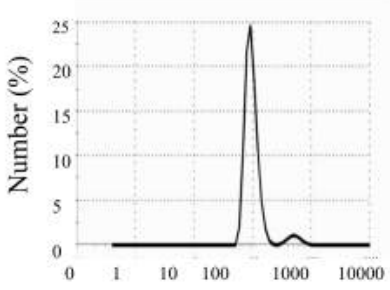

Size (d.nm)

Figure 1. Preparation and characterization of CNCs. (a) Steps of production of CNCs from the raw materials of rice straw, (b) FTIR spectrum of CNCs, (c) XRD pattern of CNCs, and (d) zeta potential and size distribution of $\mathrm{CNCs}$ nanoparticles. 


\section{Characterization of CNCs from rice straw}

\section{FTIR analysis}

IR spectrum of CNCs is shown in Figure 1b. The main bands are observed at $r 1,427 \mathrm{~cm}^{-1}$ which is assigned to a symmetric $-\mathrm{CH}_{2}$ bending vibration, as well known as "crystallinity band," while the vibration band is detected at $v 897 \mathrm{~cm}^{-1}$ which is assigned to $\mathrm{C}-\mathrm{O}-\mathrm{C}$ stretching at $\beta$ - $(1 \rightarrow 4)$-glycosidic linkages, known as "amorphous band." Moreover, the band between 2,750 and 2,900 was assigned to $\mathrm{CH}$ stretching vibration of methyl and methylene groups, while vibration bands at $r 1,372 \mathrm{~cm}^{-1}$ are assigned to $-\mathrm{CH}$ bending vibration and $r 3,314 \mathrm{~cm}^{-1}$ for $\mathrm{OH}$ stretching vibration (Thakur et al., 2020). On the other hand, peaks at $v 1,060,1,110$, and $1,162 \mathrm{~cm}^{-1}$ reveal the existence of sulfate esters which are incorporated by sulfuric acid hydrolysis reaction during CNCs' extraction process. Furthermore, the peak at $v 1,205 \mathrm{~cm}^{-1}$ is ascribed to the $\mathrm{S}=\mathrm{O}$ linkage that resulted from the esterification reaction during the preparation of CNCs ( $\mathrm{Lu}$ and Hsieh, 2010). Interestingly, the absence of bands at $v 2,850$ $\mathrm{cm}^{-1}$ and $1,732 \mathrm{~cm}^{-1}$ as displayed in CNCs spectrum is assigned to $-\mathrm{C}-\mathrm{H}$ stretching in lignin, waxes, and vibrations of acetyl and uronic ester groups of hemicelluloses or ester linkage of $-\mathrm{COOH}$ group of ferulic and $p$-coumaric acids in lignin, respectively, while the disappearance of these bands in CNCs' spectrum demonstrates that high purity of $\mathrm{CNCs}$ was obtained, which remained content free from neither lignin, hemicellulose, nor waxes (Lu and Hsieh, 2012).

\section{XRD analysis}

XRD patterns of CNCs are shown in Figure 1c. It is noted that distinctive diffraction peaks at $2 \Theta=22.5^{\circ}$ and $34.4^{\circ}$, two overlapped weaker diffraction peaks at $14.6^{\circ}$ and $16.5^{\circ}$, and a small peak at $2 \Theta=47.06^{\circ}$ are easily detected. These peaks are characteristic diffraction peaks of cellulose-I $\beta$ monoclinic structure, which usually is extracted from plants or trees, as previously described (Li and Renneckar, 2011). $\mathrm{I}_{002}$ of synthesized CNCs is of $2 \Theta=22.5^{\circ}$ and $\mathrm{I}_{\mathrm{am}}$ at peak intensity $2 \Theta=16.5^{\circ}$; thus, the crystallinity index of CNCs is almost $98 \%$. The obtained findings are consistent with the previous report by Perumal et al. (2018) who have isolated CNCs with high crystallinity index reaching $78 \%$ from rice straw. Similarly, Perumal et al. (2018) have produced $\mathrm{CNCs}$ with a crystallinity index percentage of almost $67 \%$ and $71 \%$ isolated from rice straw and rice husk, respectively. These sharp diffraction peaks of CNCs indicate that the removal of noncellulosic constituent changes the crystallinity of CNCs. During acid hydrolysis, hydronium ions of sulfuric acid attack cellulose in disordered or amorphous domains cause cleavage of glycosidic bonds and leave the crystalline domains intact. Thus, CNCs were used as reinforcing fillers in different bio-nanocomposites and biomaterials (Perumal et al., 2018). Furthermore, high $\mathrm{CrI} \%$ of extracted $\mathrm{CNCs}$ is considered an important factor for using CNCs as nanofiller in polymeric matrix providing them with high stiffness and strength.

\section{Zeta potential}

Zeta potential indicates stability and surface charge density of CNCs, which is very important during the synthesis of nanocomposites (Perumal et al., 2018). Zeta potential and size are shown in Figure 1d; it was found that the zeta potential of resultant $\mathrm{CNCs}$ is around $-24.5 \mathrm{mv}$ and this value is regarded as the minimum CNCs dispersion stability. Meanwhile, this negative charge at the surface of CNCs is corresponding to the functionalization of the surface of CNCs with sulfate groups during sulfuric acid hydrolysis treatment. Hence, an electrostatic repulsion force among nanoparticles leads to the formaton of a homogenous stable aqueous suspension ( $\mathrm{Lu}$ and Hsieh, 2012). The particle distribution of nanosize is extremely monodisperse giving clear single peak intensity with sizes range from 96 to 260 $\mathrm{nm}$ and prominent CNCs in the average size of $96 \mathrm{~nm}$, as shown in Figure 1d. The obtained size distribution of CNCs is agreed with that previously obtained during $\mathrm{CNCs}$ extraction from cotton fibers (Perumal et al., 2018).

\section{TGA thermograms}

The thermal decomposition behavior of CNCs was tested by TGA analysis, and the thermogram chart is shown in Figure 2a. Three thermal decomposition stages as weight loss versus decomposition temperature are measured. An initial weight loss of $8 \%$ is detected $\leq 100^{\circ} \mathrm{C}$ and steady-state decreases in weight up to $160^{\circ} \mathrm{C}$, owing to loss of water, solvents, or humidity evaporation. A significant weight loss in the second decomposition stage at $210^{\circ} \mathrm{C}-425^{\circ} \mathrm{C}$ is due to cellulose depolymerization. The third decomposition stage of conversion of all organic cellulose materials into carbon residues occurred after $425^{\circ} \mathrm{C}$. Approximately, $87 \%$ of the weight of $\mathrm{CNC}$ is thermally decomposed at $225^{\circ} \mathrm{C}-425^{\circ} \mathrm{C}$. In addition, a linear region of weight loss is recorded at $310^{\circ} \mathrm{C}-360^{\circ} \mathrm{C}$ which is regarded as the main characteristic thermal property of cellulose. This result was confirmed by a previous study showing the presence of the main peak move from $260^{\circ} \mathrm{C}$ to $310^{\circ} \mathrm{C}$ in the TGA curve of CNC (Thakur et al., 2020).

\section{Brunauer-Emmet-Teller (BET)}

The surface area and porosity index of CNCs were measured by the nitrogen adsorption-desorption method. BET measurement results are summarized in Table 1. The prepared CNCs possess a surface area of $\sim 181.8 \mathrm{~m}^{2} / \mathrm{g}$. The small surface area indicates that no aggregated $\mathrm{CNCs}$ were previously investigated (Abu-Danso et al., 2017). Furthermore, CNCs have a porous structure with a low average pore volume of approximately $0.1675 \mathrm{~cm}^{3} / \mathrm{g}$. The low pore volume value indicates close aligned and tight bound of CNCs via strong $\mathrm{H}-\mathrm{H}$ bonds, resulting in a little interfacial space ( $\mathrm{Lu}$ and Hsieh, 2012). Moreover, CNCs show an average pore radius $\sim 62.56 \AA$, which is slightly larger than the average pore radius of mesoporous $\mathrm{CNCs}$ extracted from rice straw (Lu and Hsieh, 2010) which was $\sim 46 \AA$, where the pores surface area of CNCs is an average of $64.617 \mathrm{~m}^{2} / \mathrm{g}$. Currently, the

Table 1. Summary of BET results of CNCs.

\begin{tabular}{cccc}
\hline Surface area $\left(\mathrm{m}^{2} / \mathrm{g}\right)$ & Average pore volume $\left(\mathrm{cm}^{3} / \mathrm{g}\right)$ & Pores surface area $\left(\mathrm{m}^{2} / \mathrm{g}\right)$ & Pores radius $(\AA)$ \\
\hline 181.8 & 0.1675 & 64.617 & $6.256 \mathrm{e}+01$ \\
\hline
\end{tabular}


obtained results of BET are similar to those obtained from CNCs extracted from rice straw with very low cumulative pore volumes ( $\sim 0.025$ and $\left.0.084 \mathrm{~cm}^{3} / \mathrm{g}\right)$ (Lu and Hsieh, 2012).

\section{TEM photographs}

TEM images of isolated CNCs show that they possess uniform distributed and nonaggregated rod-like or needle-like structures, as shown in Figure $2 \mathrm{~b}$. The individual particles are characterized by homogeneity and nanometric dimensions as the average diameter of nanocrystals, which ranged from $\sim 20$ to 60 $\mathrm{nm}$ in diameter, with length ranged from $\sim 250$ to $480 \mathrm{~nm}$ through size distribution diagrams of prepared CNCs. Such results are consistent with that previously published dimensions which were at $30.7 \mathrm{~nm}$ in diameter and $270 \mathrm{~nm}$ in length (Lu and Hsieh, 2012).

\section{Preparation and optimization of PVA/HA/CNCs composite hydrogel membranes}

Crosslinked PVA/HA hydrogel membranes were prepared by the solution-casting method using different ratios of PVA:HA as 80:20, 60:40, and 50:50, while CA was added as a chemical crosslinker. The optimized volume ratio of PVA:HA (80:20) and $10 \%$ of CA were used for further experiments; the obtained membranes were thermally treated at $80^{\circ} \mathrm{C}$ for 18

Thermogravimetric analysis of $\mathrm{CNCs}$
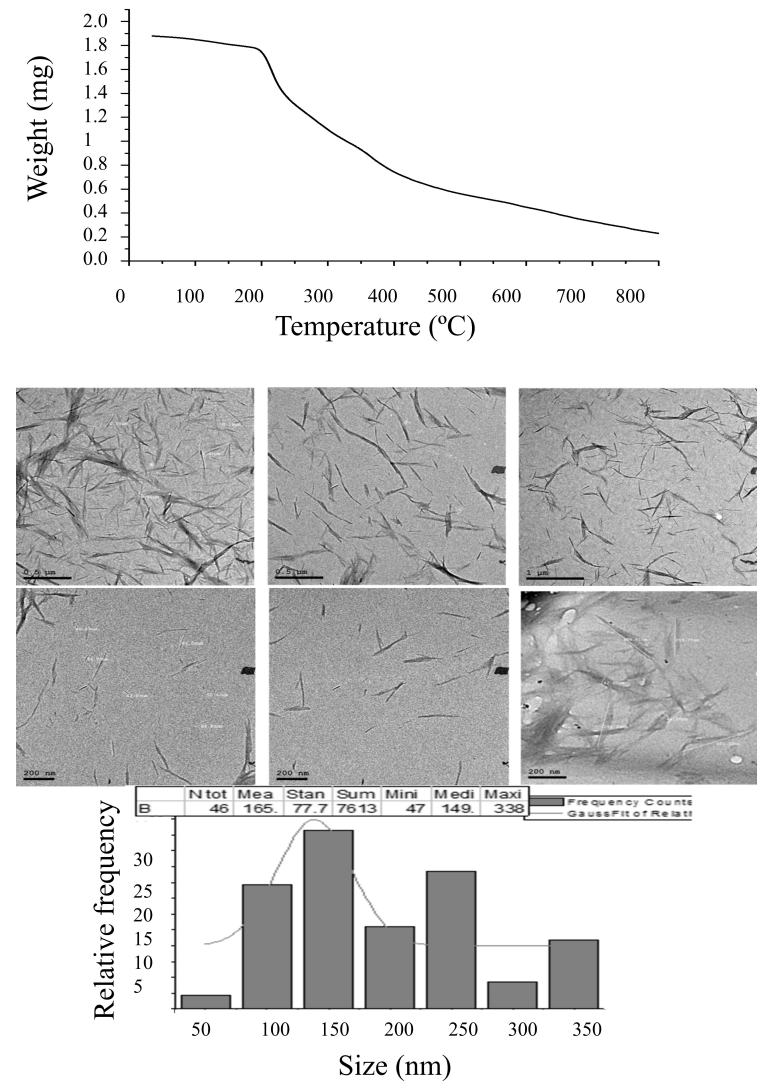

Figure 2. (a) TGA thermograph of CNCs and (b) TEM images of CNCs nanoparticles. hours for initiating the crosslinking process. Also, CNCs were incorporated into PVA/HA in different ratios $(0.25 \%, 0.5 \%$, and $1.0 \%, w / v)$ for testing crosslinked PVA/HA/CNCs hydrogel membrane mechanical improvement.

\section{Characterization of PVA/HA/CNCs composite hydrogel membranes}

\section{SEM photographs}

SEM photographs of crosslinked PVA/HA/CNCs composite hydrogel membranes with different ratios of $\mathrm{CNCs}$ $[0 \%, 0.25 \%, 0.5 \%$, and $1.0 \%(w / v)]$ are shown in Figure 3. PVA/HA hydrogel membranes have very smooth, uniform, compacted, and roughed surfaces membranes as shown in Figure 3. Moreover, randomly, few, small, scattered, and spherical pores might be formed during heat treatment (Rahman et al., 2019). Previously, Xue et al. (2020) observed the formation of cracked 1,4-butanedioldiglycidy/HA hydrogels crosslinked with different molecular weights of HA. After incorporation of $\mathrm{CNCs}$ at a concentration of $0.5 \%$, the obtained hydrogel membranes were homogeneous, compacted, dense, and uniform with a smooth surface; this might be attributed to the proper dispersion of $\mathrm{CNCs}$ into PVA/HA matrix. In addition, homogeneity improvement in membrane might be related to changing the orientation of PVA

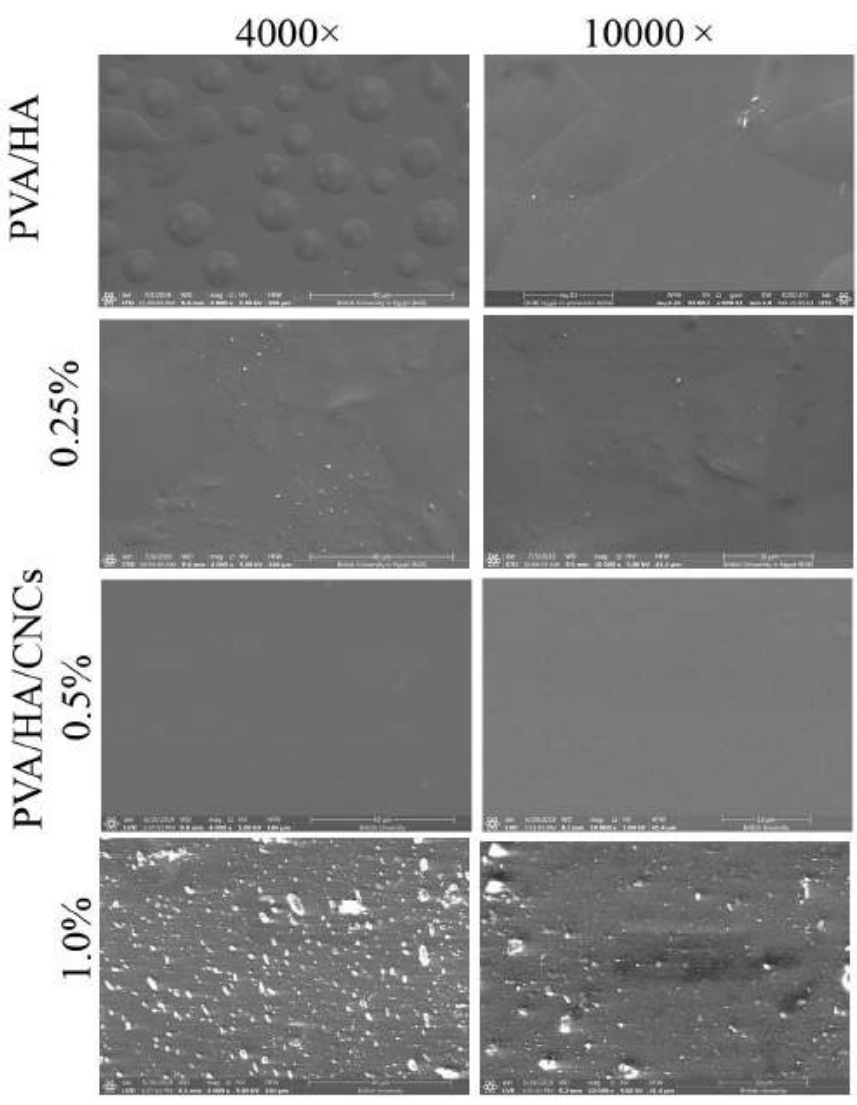

Figure 3. SEM photographs of PVA/HA/CNCs hydrogel membranes with different contents of CNCs $(0,0.25,0.5$, and $1 \%)$ at original magnifications $(4,000 \times$ and $10,000 \times)$ at $5 \mathrm{Kv}$. 
and $\mathrm{HA}$ chains, thus exposing abundant $-\mathrm{OH}$ group to form intermolecular hydrogen bonding which enhances the miscibility of PVA and HA and with each other (Uranga et al., 2020). On the contrary, SEM images with a high content of CNCs (1.0\%) show rough, nonuniform, and cracked hydrogel membranes. In addition, the SEM photograph indicates the appearance of white dots on the PVA/HA membrane, which correspond to the aggregation of $\mathrm{CNCs}$ in the interior structure of the membrane.

\section{FTIR analysis}

Figure 4a shows the FTIR spectra of PVA, HA, CNCs, uncrosslinked, crosslinked PVA/HA, and crosslinked PVA/ HA/CNCs hydrogel membranes. It is clearly observed that characteristic bands of PVA are detected at $v 3,289,2,903$, and $1,713 \mathrm{~cm}^{-1}$ which correspond to hydroxyl, alkyl, and acetyl groups, respectively (Kumar and Han, 2017). Meanwhile, HA shows bands at $v 1,683 \mathrm{~cm}^{-1}$ and $1,559 \mathrm{~cm}^{-1}$ which correspond to the vibration of $\mathrm{C}=\mathrm{O}$ in the amide II and bending vibration of amide II N-H. A band at $v 3,340 \mathrm{~cm}^{-1}$ is ascribed to $-\mathrm{NH}$ and -OH stretching vibrations. Also, a sharp band at $v 2,922 \mathrm{~cm}^{-1}$ is due to the $\mathrm{C}-\mathrm{H}$ peak, while two absorption bands at $v 1,030$ $\mathrm{cm}^{-1}$ and $1,265 \mathrm{~cm}^{-1}$ are corresponding to $\mathrm{C}-\mathrm{O}-\mathrm{C}$ symmetric and asymmetric vibration band (Yin et al., 2019), while CNCs have main bands at $v 1,427 \mathrm{~cm}^{-1}$ which is assigned to a symmetric $-\mathrm{CH}_{2}$ bending vibration, well-known as the "crystallinity band," while

a

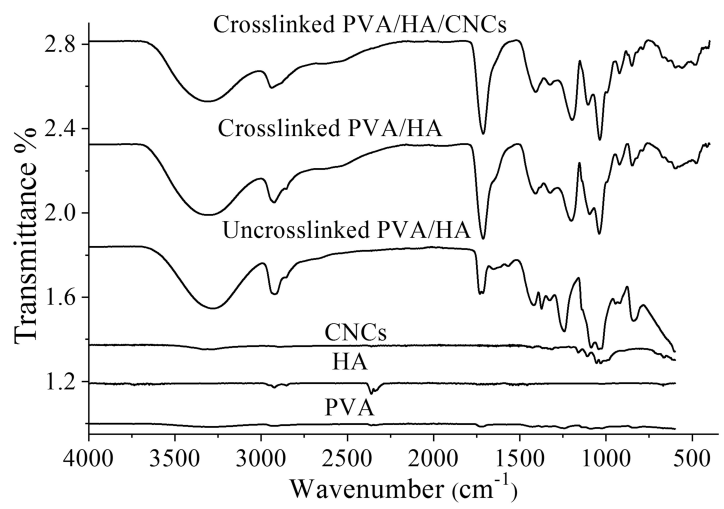

$\mathrm{b}$

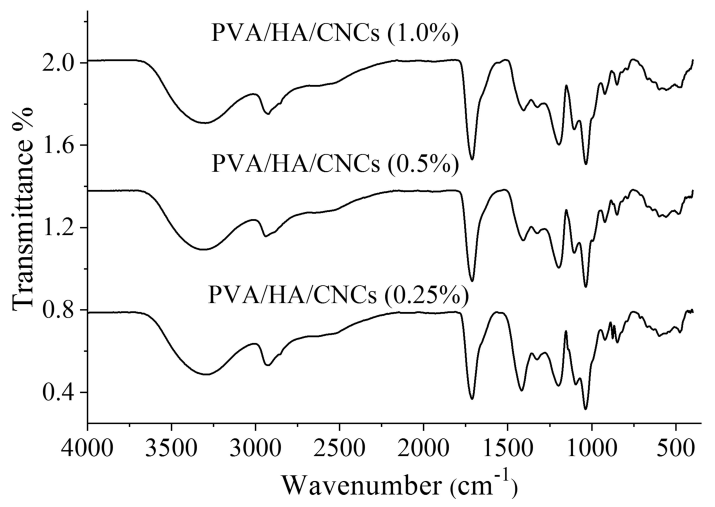

Figure 4. FTIR spectra of (a) PVA, HA, CNCs, uncrosslinked PVA/HA membrane, crosslinked PVA/HA membrane, and crosslinked PVA/HA/CNCs hydrogel membranes and (b) PVA/HA/CNCs with different contents of $\mathrm{CNCs}$ $(0.25,0.5$, and $1.0 \%)$. the peak at $897 \mathrm{~cm}^{-1}$ is assigned to $\mathrm{C}-\mathrm{O}-\mathrm{C}$ stretching vibration of $\beta$ - $(1 \rightarrow 4)$-glycosidic linkages, known as the "amorphous band." Moreover, vibration bands at 1,372 and 2,600 $\mathrm{cm}^{-1}$ are assigned to $-\mathrm{CH}$ bending vibration and $v 3,314 \mathrm{~cm}^{-1}$ for $-\mathrm{OH}$ stretching vibration (Thakur et al., 2020). Furthermore, peaks at 1,060, 1,110, 1,162 , and $1,205 \mathrm{~cm}^{-1}$ demonstrate the existence of sulfate esters which are functionalized $\mathrm{CNCs}$ during the sulfuric acid hydrolysis process (Lu and Hsieh, 2010).

For uncrosslinked PVA/HA, two polymers interact with intermolecular hydrogen bonds through their terminal $\mathrm{OH}$ moieties. This is evidenced by reducing the intensity and the broadening of the $-\mathrm{OH}$ band in PVA, blue shift into higher wavenumber from $3,288 \mathrm{~cm}^{-1}$ to $3,314 \mathrm{~cm}^{-1}$, compared to pure PVA. Additionally, a strong shift of $\mathrm{C}-\mathrm{O}$ band in HA from 1,265 $\mathrm{cm}^{-1}$ to $1,399 \mathrm{~cm}^{-1}$ is observed, suggesting the formation of $\mathrm{H}-\mathrm{H}$ bonds between HA and PVA. This observation was also reported during the preparation of the hyaluronan/PVA-SbQ composite film (Xu et al., 2013).

After crosslinking, a new peak is found within the spectral region particularly at $v 1,725 \mathrm{~cm}^{-1}$. This peak is owing to $\mathrm{C}=\mathrm{O}$ of ester groups formed between $-\mathrm{COOH}$ groups of $\mathrm{CA}$ acid and $-\mathrm{OH}$ of PVA. CA could react with $-\mathrm{OH}$ of two separate PVA chains or with $-\mathrm{OH}$ of the same chain-forming intermolecular or intramolecular crosslinking; this is dependent on the orientation of CA and polymer chains (Li et al., 2018). Thus, the band corresponded to the $-\mathrm{OH}$ group shifted from 3,314 to $3,305 \mathrm{~cm}^{-1}$. The mechanism of esterification crosslinking is preceded through an equilibrium-controlled reaction between $-\mathrm{COOH}$ group in $\mathrm{CA}$ and $-\mathrm{OH}$ group in PVA. The reaction starts when a reactive cyclic anhydride is formed by heating and dehydrating CA which in turn reacts with-OH groups in PVA (do Nascimento et al., 2020).

After introducing $\mathrm{CNCs}$ into the PVA/HA membrane, the characteristic peaks of CNCs appear, such as glycoside linkage and sulfate ester groups. It is observed that an increase in the peak intensity belongs to stretching vibration of aliphatic moieties of polysaccharides as $-\mathrm{OH}$ around $v 3,302 \mathrm{~cm}^{-1}$ and $-\mathrm{CH}$ at $r 2,927 \mathrm{~cm}^{-1}$. Moreover, it is detached that $-\mathrm{OH}$ peak is shifted into a lower wavenumber compared to $-\mathrm{OH}$ of free $\mathrm{CNCs}$. This might be a strong $\mathrm{H}-\mathrm{H}$ interaction among CNCs, PVA, and HA. Furthermore, it is noted that the stretching band of $\mathrm{C}-\mathrm{O}-\mathrm{C}$ shifted from $v 898$ to $878 \mathrm{~cm}^{-1}$, suggesting possible interactions between PVA and CNCs.

Increasing intensity at $v 1,725 \mathrm{~cm}^{-1}$ in response to increasing concentration of CNCs is displayed, as shown in Figure $4 \mathrm{~b}$. This band has corresponded to ester carbonyl group $(\mathrm{C}=\mathrm{O})$ group stretching vibration since some of the $\mathrm{CNCs}$ are crosslinked by $\mathrm{CA}$. Moreover, $-\mathrm{OH}$ band is increased in intensity and is shifted into lower wavenumber with an increasing percentage of $\mathrm{CNCs}$, compared to $\mathrm{OH}$ of free CNCs.

\section{Mechanical testing}

Mechanical properties of crosslinked PVA/HA/CNCs composite hydrogel membranes with different contents of $\mathrm{CNCs}$ $(0 \%, 0.25 \%, 0.5 \%$, and $1.0 \%, w / v)$, for example, Young's modulus, elastic modulus, elongation at break, maximum force, maximum stress, and maximum displacement, were investigated in the wet state of membranes, as shown in Table 2. As can be seen, the mechanical performance of crosslinked PVA/HA membranes 
is improved significantly with the introduction of CNCs into membrane composition, where the mechanical parameters of crosslinked PVA/HA/CNCs composite hydrogel membrane with $0.5 \%$ of $\mathrm{CNCs}$ were increased progressively, compared to that without CNCs (Table 2). The reinforcement effect was attributed to the high aspect ratio of $\mathrm{CNCs}$ enabling the formation of loadbearing structure through the hydrogen bonding interaction with other polymeric chains. In addition, strong electrostatic interactions are between the negatively charged sulfate groups of CNCs and the cationic amine of HA (Hu et al., 2020).

On the contrary, the mechanical parameters of crosslinked PVA/HA/CNCs composite membranes with $1.0 \%$ of $\mathrm{CNCs}$ are lower than composite membranes with $0.5 \% \mathrm{CNCs}$; however, they are higher than membranes without CNCs. This phenomenon was attributed to the fact that $\mathrm{CNCs}$ aggregation and viscosity increased by increasing the concentration of CNCs up to $0.5 \%$. Thereafter, nonhomogenous dispersion of CNCs in the membrane resulted in inefficient stress transfer between PVA/HA matrix and $\mathrm{CNCs}$ and decreasing the mechanical properties of the membrane. A similar result was reported through the preparation of PVA/chitosan film reinforced with varied contents of CNCs (Perumal et al., 2018).

\section{XRD analysis}

Figure 5 shows the XRD patterns of $\mathrm{CNCs}$, crosslinked PVA/HA, and crosslinked PVA/HA/CNCs hydrogel membranes with ranged amounts of CNC of $0 \%, 0.25 \%, 0.5 \%, 1.0 \%(w / v)$. The appearance of diffraction patterns of CNCs at $2 \Theta=22.5^{\circ}$, $34.4^{\circ}, 14.6^{\circ}, 16.5^{\circ}$, and $47.06^{\circ}$ is found easily. These characteristic diffraction patterns are corresponding to the crystal structure of cellulose-I which is isolated from a plant source, as previously described by Li and Renneckar (2011), while crosslinked PVA/ HA membranes exhibit semicrystalline diffraction patterns of PVA at $2 \Theta=19.5^{\circ}$ and $40.7^{\circ}$. Furthermore, the blending of PVA with HA shift peaks of PVA at $2 \theta=22.3^{\circ}$ and $40.3^{\circ}$ into $2 \Theta=$ $20.4^{\circ}$ and $37.3^{\circ}$. In the case of PVA/HA/CNC membrane, the appearance of the distinctive peak of CNCs at $2 \Theta=14.6^{\circ}-34.7^{\circ}$ is easily detected, indicating the successful incorporation of $\mathrm{CNCs}$ into PVA/HA membrane. By increasing $\mathrm{CNC}$ content to $0.5 \%$ in $\mathrm{PVA} / \mathrm{HA} / \mathrm{CNCs}$ membrane, peak at $2 \Theta=22.5^{\circ}$ become sharper than revealing the efficient incorporation and interaction of $\mathrm{CNC}$ in PVA/HA membranes. Then, this peak is reduced when CNCs amount was further increased to $1.0 \%$. Similarly, Souza Coelho et al. reported that the peaks intensity of CNCs increases with an increase in $\mathrm{CNC}$ content in the starch-based biocomposite film (de
Souza Coelho et al., 2020). Moreover, CrI of all membranes was calculated by the Segal equation, since CrI has a strong influence on the stability of biocomposite membranes. CrI of PVA/HA membrane increases from $47 \%$ to $50 \%$, after introducing $\mathrm{CNCs}$ at a concentration of $0.25 \%$ in the membrane. It is observed that a $\mathrm{CrI}$ of PVA/HA/CNCs membrane is directly proportional to the mass of introduced $\mathrm{CNCs}$, till $0.5 \%$, whereas $\mathrm{CrI}$ of PVA/HA/ CNCs of $0.25 \%$ and $0.5 \%$ CNCs is $50 \%$ and $98 \%$, respectively. Consequently, $\mathrm{CrI}$ of PVA/HA/CNCs is strongly reduced to $28.8 \%$ by increasing $\mathrm{CNCs}$ concentration to $1.0 \%$ in the composite hydrogel membranes. This reduction of $\mathrm{CrI}$ gets along with a decrease in mechanical performance as presented in Table 2, at the same CNCs content owing to the aggregation behavior of CNCs.

\section{TGA thermographs}

TGA of composite membranes was measured according to our previously published study; PVA/HA/CNCs membrane has exceeded the thermal stability of PVA/HA membrane owing to the incorporation of CNCs (Hussein et al., 2020), where PVA/ $\mathrm{HA} / \mathrm{CNCs}$ composite membranes were thermally decomposed of $\mathrm{T}_{\text {onset }}$ at $202^{\circ} \mathrm{C}$ and second decomposition stage at $260^{\circ} \mathrm{C}-365^{\circ} \mathrm{C}$, compared to PVA/HA membrane at $\mathrm{T}_{\text {onset }}$ at $190^{\circ} \mathrm{C}$ and second decomposition stage at $190^{\circ} \mathrm{C}-340^{\circ} \mathrm{C}$, due to the absence of CNCs (Hussein et al., 2020).

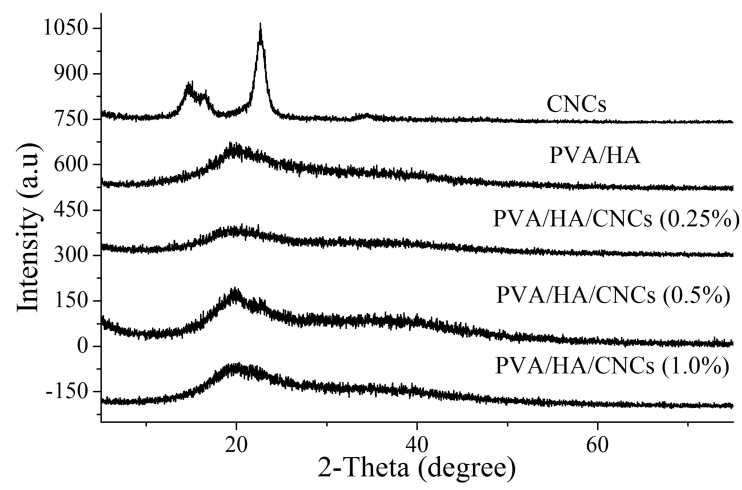

Figure 5. XRD patterns of $\mathrm{CNCs}$ and crosslinked $\mathrm{PVA} / \mathrm{HA} / \mathrm{CNCs}$ with varied CNCs contents $(0,0.25,0.5$, and $1 \%)$.

Table 2. Mechanical properties of crosslinked PVA/HA and PVA/HA/CNCs composite hydrogel membranes with varied contents of CNCs $(0$, $0.25,0.5$, and $1.0 \%, w / v)$.

\begin{tabular}{|c|c|c|c|c|c|c|c|}
\hline Membrane composition & $\begin{array}{c}\text { CNCs } \\
\text { content } \\
(\%, w / v)\end{array}$ & $\begin{array}{c}\text { Max. } \\
\text { force } \\
(N)\end{array}$ & $\begin{array}{l}\text { Max. stress } \\
\left(\mathrm{N} / \mathbf{m m}^{2}\right)\end{array}$ & $\begin{array}{c}\text { Elongation at break } \\
(\%)\end{array}$ & $\begin{array}{l}\text { Max. displacement } \\
(\mathrm{mm})\end{array}$ & $\begin{array}{c}\text { Young's } \\
\text { modulus (MPa) }\end{array}$ & $\begin{array}{c}\text { Elastic } \\
\text { modulus }(N / \\
\left.\text { mm }^{2}\right) \\
\end{array}$ \\
\hline $\mathrm{PVA} / \mathrm{HA}$ & 0 & 20 & 13 & 327 & 57 & 187 & 1.46 \\
\hline $\mathrm{PVA} / \mathrm{HA} / \mathrm{CNCs}$ & 0.25 & 24 & 18 & 385 & 56 & 218 & 1.66 \\
\hline $\mathrm{PVA} / \mathrm{HA} / \mathrm{CNCs}$ & 0.5 & 48 & 30 & 635 & 85 & 277 & 2.21 \\
\hline $\mathrm{PVA} / \mathrm{HA} / \mathrm{CNCs}$ & 1.0 & 24 & 17 & 420 & 61 & 220 & 1.91 \\
\hline
\end{tabular}

All tested hydrogel membranes contain PVA:HA in the ratio of 80:20, crosslinked with $10 \% \mathrm{CA}$. 


\section{Physicochemical properties of PVA/HA/CNCs composite hydrogel membranes}

\section{Swelling (\%)}

The swelling ratio of hydrogel membranes was tested for 14 days, as shown in Figure 6a. Crosslinked PVA/HA hydrogel membrane and its composite of PVA/HA/CNCs with different CNCs contents reached an equilibrium swelling state between 8 and 14 days. Interestingly, composite hydrogel membranes with $0.5 \%$ of CNCs reached the equilibrium swelling after 8 days, with the highest swelling ratio of $\sim 1,200 \%$, whereas PVA/HA hydrogel membrane with $0 \% \mathrm{CNCs}$ reached an equilibrium swelling state after 9 days, with a swelling ratio of $\sim 600 \%$. This implies that CNCs' incorporation significantly enhanced the swelling behavior of PVA/HA hydrogel membranes. This might be owing to the hydrophilic nature of HA and CNCs that increases the formation of hydrogen bonds accompanied by a marked increase in water absorption. Moreover, CNCs could interact with water molecules owing to their large number of free $-\mathrm{OH}$ and $\mathrm{SO}_{3} \mathrm{H}$ groups resulting from their high surface area. Recently, Yin et al. (2019) reported a similar effect of $\mathrm{CNCs}$ in $\mathrm{CNCs}$ onto gelatin/HA hydrogel. Similarly, the addition of $0.25 \% \mathrm{CNCs}$ into composite membranes enhanced the swelling ratio to $925 \%$, compared to $0 \%$ of CNCsPVA/HA hydrogel membranes which produced a swelling ratio

a

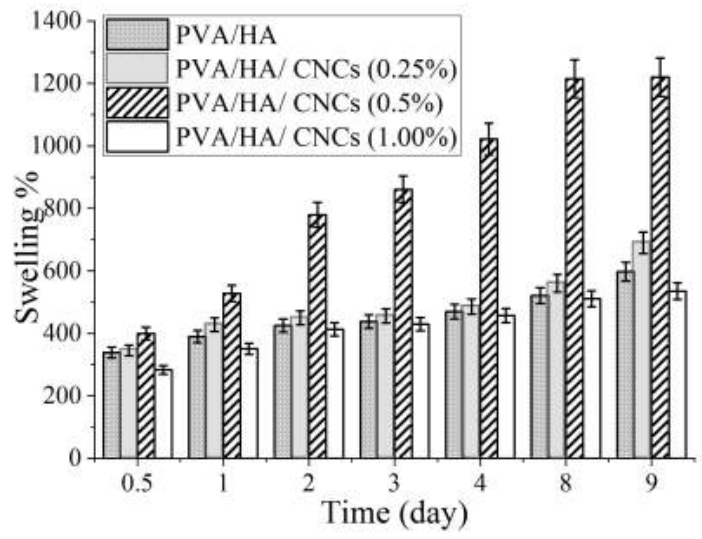

b

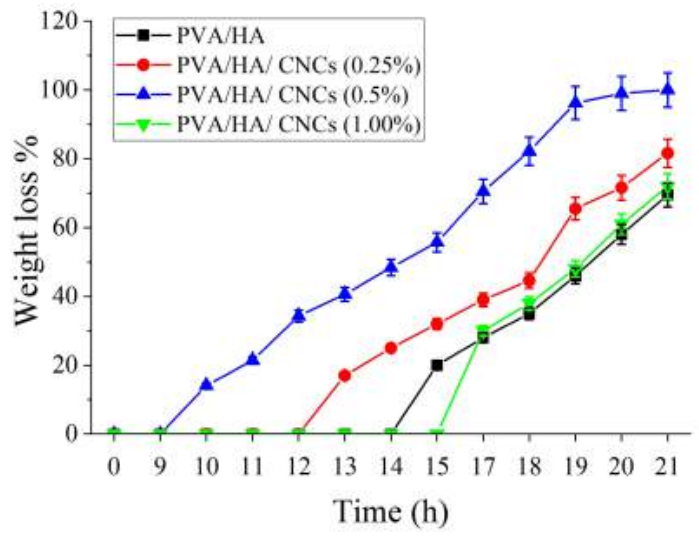

Figure 6. (a) Swelling ratio (\%) and (b) hydrolytic degradation as weight loss (\%) of crosslinked PVA/HA/CNCs composite hydrogel membranes of various CNCs contents $(0,0.25,0.5$, and $1 \%)$. of $825 \%$ after 12 days for each other. Unexpectedly, such a ratio is reduced to $\sim 500 \%$ by increasing the concentration of CNCs to $1.0 \%$ after the same period (12 days) compared to the membrane without CNCs. This is attributed to strong intermolecular hydrogen bonds in CNCs that obstacle water diffusion into the membrane. Furthermore, excess CNCs might lead to aggregation and nonhomogenous dispersion of CNCs which in turn decreased swelling capacity (Xu et al., 2018).

\section{Hydrolytic degradation}

Hydrolytic degradation of tested membranes was evaluated by immersion of composite membranes into PBS for 21 days, as shown in Figure 6b. As expected, PVA/HA hydrogel membranes lose about $25 \%$ of its weight on day 15 and $50 \%$ on day 19, respectively. It is owing to the slow dissolution of PVA and quick hydrolytic degradation then releasing of PVA chains that cannot participate in the crystallite domains. Furthermore, a secession of crosslinker segments that binds polymers chains might occur, followed by decomposition of crosslinked polymeric chains (Fahmy et al., 2015). However, it was observed that composite hydrogel membranes with different CNCs contents $(0.25 \%, 0.5 \%$, and $1.0 \%, w / v)$ lose $25 \%$ of their weight loss on days 12,9 , and 15 , and $50 \%$ of their weight loss on days 19,15 , and 20, respectively. This implies that the incorporation of CNCs into PVA/HA composite hydrogel membranes might slightly prolong the duration of hydrolytic degradation, particularly in the last stages. Conversely, PVA/HA hydrogel membranes without CNCs seem to be hydrolytically more stable than that containing CNCs, particularly after 15 days of immersion. This is ascribed to the impact of CNCs on enhancing hydrophilicity which permits water diffusion and decreases crosslinking density in the membrane; however, the higher amount of CNCs $(1.0 \%)$ exhibits the lower degradation rate. This observation is owing to the crystalline property of CNCs polymer which facilitates its rapid degradation. In addition, CNCs are crystalline polymer; hence, increasing CNCs content perhaps increased crystalline regions that are degraded much slowly. Therefore, PVA/HA/CNCs with $0.5 \%$ CNCs were chosen as appropriate degradation rate for further bioevaluation experiments and for therapeutically wound dressing materials.

\section{Bioevaluation assays of $\mathrm{CNCs}$ and crosslinked PVA/HA/ CNCs composite hydrogel membranes}

\section{Cytotoxicity test}

The cytotoxicity of prepared CNCs was evaluated by MTT assay at various concentrations of CNCs on HFB-4 and WI38 human cell lines. Results revealed that our prepared CNCs are not toxic on both tested cell lines (Fig. 7a). However, a high concentration of CNCs (i.e., $\geq 1,000 \mu \mathrm{g} / \mathrm{ml}$ ) reduces slightly HFB-4 and WI38 cell viability. Such observation is due to an increase in the cellular uptake of treated materials causing cell damage mediated by expression of oxidative stress and inflammatory cytokines (Menas et al., 2017). These results are consistent with that previously reported that $\mathrm{CNCs}$ of various sizes at high concentrations of 500 and $1,000 \mu \mathrm{g} / \mathrm{ml}$ induced very slight cytotoxicity in NIH3T3 murine embryo fibroblast cells and HCT116 colon adenocarcinomas; they explained that the exerted cytotoxic effect was due to the formation 

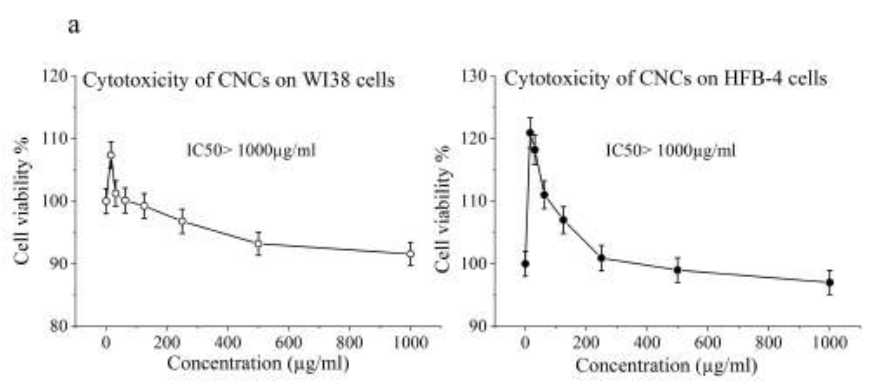

b

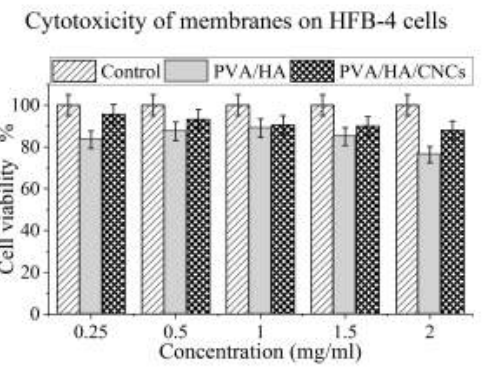

Figure 7. In vitro cell viability of (a) CNCs on WI38 and HFB-4 cell lines and (b) on crosslinked PVA/HA and PVA/HA/CNCs of $0.5 \%$ CNCs of composite hydrogel membranes on $H F B-4$ cell lines, as a function of different membranes' concentrations $(0.25,0.5,1.0,1.5$, and $2.0 \mathrm{mg} / \mathrm{ml})$.

of gel suspension that blocked the passage of gases through the cytoplasmic membrane (Hanif et al., 2014).

MTT assay of crosslinked PVA/HA and PVA/HA/CNCs was carried out using HFB-4 cell lines (Fig. 7b). Compared to control cells after 48 hours of cell exposure, all membranes exhibited a nontoxic effect onto HFB-4 cells; that means cells continuously grow and proliferate on membranes, as shown in Figure 7b. It was observed that crosslinked PVA/HA/CNCs membranes possess higher cell viability (\%) than crosslinked PVA/ HA membranes, especially at concentrations of 0.25 and $2(\mathrm{mg} /$ $\mathrm{ml})$. Since PVA/HA/CNCs membrane recorded cell viability (\%) of $\sim 96 \%$ and $83 \%$ versus $\sim 84 \%$ and $76 \%$ of PVA/HA membrane at concentration 0.25 and $2 \mathrm{mg} / \mathrm{ml}$, respectively, the high cell viability mechanism might arise from the biomechanical signal of CNCs that can enhance the physiological function of HA in cell growth through stimulation of collagen formation and epithelial cell proliferation increased (Singla et al., 2017).

\section{Hemocompatibility test}

The hemolysis test was conducted to investigate the ability of CNC powder to rupture the tested RBCs and liberate its hemoglobin content. Figure 8 a reflects the biocompatibility degree of CNCs particles and tested membranes, compared to the positive control. The degree of blood hemolysis was varied according to the tested materials. CNCs powder, crosslinked PVA/HA, and $\mathrm{PVA} / \mathrm{HA} / \mathrm{CNCs}$ composite hydrogel membranes show an optical density of $0.967,0.862$, and 1.165 , which are very close and compared to 1.4 for positive control. These results indicate that CNCs powder is moderately safe against human cells. Notable, composite hydrogel membranes containing $0.5 \%$ CNCs exhibited considerable haemocompatibility compared to the positive control and other competitive materials.

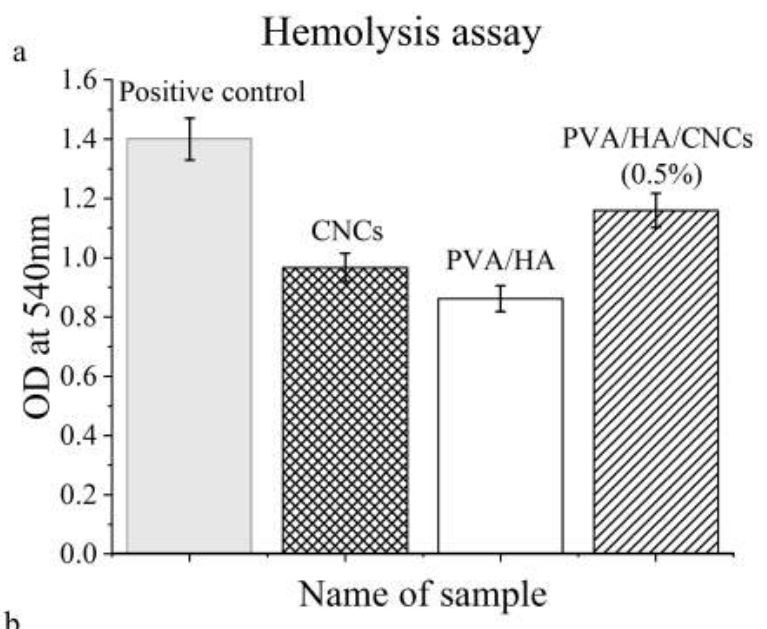

Antimicrobial activity of membranes

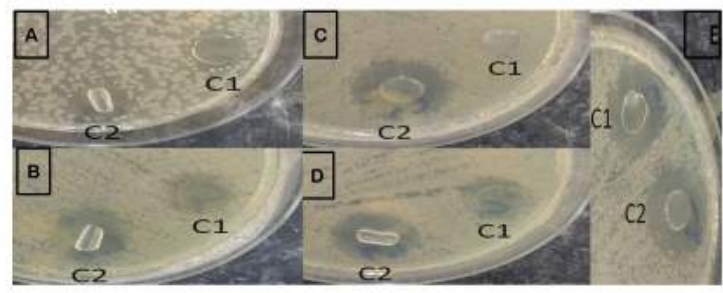

Figure 8. (a) Hemolysis test of $\mathrm{CNCs}, \mathrm{PVA} / \mathrm{HA}$ crosslinked membrane, and $\mathrm{PVA} / \mathrm{HA} / \mathrm{CNCs}(0.5 \%)$ crosslinked membrane. (b) Antimicrobial activity of $\mathrm{PVA} / \mathrm{HA}$ and PVA/HA/CNCs of $0.5 \%$ CNCs crosslinked hydrogel membranes.

\section{Antimicrobial activity}

Table 3 and Figure $8 \mathrm{~b}$ show the ability of isolated CNCs and tested membranes to resist the growth of pathogenic bacteria and yeast using disk diffusion assay. It is demonstrated that CNCs powder fails to stop the growth of five tested pathogenic microbes, as indicated by the absence of clear zones after the incubation period against all pathogenic microbes. Thus, CNCs did not show any antimicrobial activity against tested microbes; these results are consistent with Bhat et al. (2017). Upon incorporation of CNCs into PVA/HA membrane, it exhibits strong antimicrobial activity against all tested pathogens evidenced by clear inhibition zones ranging from 12 to $21 \mathrm{~mm}$. The antimicrobial activity of HA is promoted by inhibiting adhesion between cell's receptor and microorganism forming a pericellular jelly barrier surrounding the cells (Fallacara et al., 2018). The formed inhibition zone around the disk of PVA/HA with CNCs might be related to the hydrophilic nature of CNCs. This is because the hydrophilicity of microbes and substrate is a key parameter for controlling the sensitivity of the antimicrobial agent of the tested material. A similar effect was obtained in the case of preparing chitosan/CNCs tissue paper (Tyagi et al., 2018). Our prepared PVA/ HA hydrogel membranes exhibited antimicrobial activity against $V$. cholerae, C. albicans, K. pneumonia, E. coli, and K. pneumoniae with inhibition zones at 11, 14, 17, and $23 \mathrm{~mm}$, respectively, and for PVA/HA/CNCs at 12, 19, 19, and $21 \mathrm{~mm}$, respectively. Regarding, $\mathrm{PVA} / \mathrm{HA} / \mathrm{CNCs}$ membrane, it inhibits the most resistant pathogen, $P$. aeruginosa, with an inhibition zone at $21 \mathrm{~mm}$, compared to PVA/HA membranes which do not show such activity. 
Table 3. Antimicrobial activity of CNCs, crosslinked PVA/HA, and PVA/HA/ CNCs hydrogel membranes against pathogenic bacteria and yeast.

\begin{tabular}{lccc}
\hline \multirow{2}{*}{ Microbial pathogens } & \multirow{2}{*}{ CNCs } & \multicolumn{2}{c}{ Inhibition clear zone $(\mathbf{m m})$} \\
\cline { 3 - 4 } & & PVA/HA & PVA/HA/CNCs \\
\hline V. cholerae & 0 & 11 & 12 \\
C. albicans & 0 & 14 & 19 \\
P. aeruginosa & 0 & 0 & 21 \\
E. coli & 0 & 17 & 19 \\
K. pneumoniae & 0 & 23 & 21 \\
\hline
\end{tabular}

\section{CONCLUSION}

In summary, this study represents an alternative pathway of disposal rice straw instead of burning process, through converting rice straw pulp into CNCs biocomposite for production of low cost wound dressing materials. Generally, CNCs were successfully extracted from rice straw using the alkaline-acid hydrolysis treatment process. Herein, CNCs were incorporated into PVA/HA hydrogel membranes for improving the entire hydrogel membranes properties. Incorporation of CNCs into crosslinked PVA/HA composite hydrogel membranes enhanced significantly mechanical/thermal properties of membranes, increased swelling ratio, and slight stability against hydrolytic degradation. Both prepared CNCs and crosslinked $\mathrm{PVA} / \mathrm{HA} / \mathrm{CNCs}$ composite hydrogel membranes were not toxic on human cell lines. PVA/HA/CNCs composite hydrogel membranes showed higher hemocompatibility and antimicrobial activity than CNCs or PVA/HA hydrogel membranes. Thus, PVA/ $\mathrm{HA} / \mathrm{CNCs}$ could be taken in consideration as good and promised biomaterial candidate for biomedical applications particularly for wound dressing materials.

\section{ACKNOWLEDGMENTS}

Y. Hussein, Ahmed I. Abdel-Salam, and A. S. Mansour thank the partial financial support through a project of full integration of rice husk and straw for producing nanosilica and nanocellulose for deepening the local industries.

\section{AUTHOR CONTRIBUTIONS}

All authors made substantial contributions to conception and design, acquisition of data, or analysis and interpretation of data; took part in drafting the article or revising it critically for important intellectual content; agreed to submit to the current journal; gave final approval of the version to be published; and agree to be accountable for all aspects of the work.

\section{FUNDING}

There is no funding to report.

\section{CONFLICTS OF INTEREST}

The authors report no conflicts of interest in this work.

\section{ETHICAL APPROVAL}

This study does not involve the use of animals or human subjects.

\section{REFERENCES}

Abu-Danso E, Srivastava V, Sillanpää M, Bhatnagar A Pretreatment assisted synthesis and characterization of cellulose nanocrystals and cellulose nanofibers from absorbent cotton. Int J Biol Macromol, 2017; 102(2017):248-57.
Bespalova Y, Kwon D, Vasanthan N. Surface modification and antimicrobial properties of cellulose nanocrystals. J Appl Polym Sci, 2017; 134(18):1-7.

Bhat AH, Dasan Y, Khan I, Soleimani H, Usmani A. Application of nanocrystalline cellulose: processing and biomedical applications. Cellulose-reinforced nanofibre composites: Elsevier, Amsterdam, Netherlands, pp 215-40, 2017.

Dehkordi NK, Minaiyan M, Talebi A, Akbari V, Taheri A. Nanocrystalline cellulose-hyaluronic acid composite enriched with GMCSF loaded chitosan nanoparticles for enhanced wound healing. Biomed Mater, 2019; 14(3):1-51.

de Souza Coelho CC, Silva RBS, Carvalho CWP, Rossi AL, Teixeira JA, Freitas-Silva O, Cabral LMC. Cellulose nanocrystals from grape pomace and their use for the development of starch-based nanocomposite films. Int J Biol Macromol, 2020; 159:1048-61.

do Nascimento FC, de Aguiar LCV, Costa LAT, Fernandes MT, Marassi RJ, de Souza Gomes A, de Castro JA. Formulation and characterization of crosslinked polyvinyl alcohol (PVA) membranes: effects of the crosslinking agents. Polym Bull, 2020; 2020:1-13.

Du H, Liu W, Zhang M, Si C, Zhang X, Li B. Cellulose nanocrystals and cellulose nanofibrils based hydrogels for biomedical applications. Carbohydr Polym, 2019; 209: 130-44.

El-Dewany C, Zaghloul A, Awad F. Utilization of rice straw as a low-cost natural by-product in agriculture. Int J Environ Pollut Environ Model, 2018; 1(4):91-102.

El Miri N, Abdelouahdi K, Zahouily M, Fihri A, Barakat A, Solhy A, El Achaby M. Bio-nanocomposite films based on cellulose nanocrystals filled polyvinyl alcohol/chitosan polymer blend. J Appl Polym Sci, 2015; 132(22):1-13.

Fahmy A, Kamoun EA, El-Eisawy R, El-Fakharany EM, Taha TH, El-Damhougy BK, Abdelhai F. Poly (vinyl alcohol)-hyaluronic acid membranes for wound dressing applications: synthesis and in vitro bioevaluations. J Braz Chem Soc, 2015; 26(7):1466-74.

Fallacara A, Baldini E, Manfredini S, Vertuani S. Hyaluronic acid in the third millennium. Polymers, 2018; 10(7):701-36.

Ghorbani M, Roshangar L, Rad JS. Development of reinforced chitosan/pectin scaffold by using the cellulose nanocrystals as nanofillers: an injectable hydrogel for tissue engineering. Eur Polym J, 2020; 130:1-9.

Hadisi Z, Farokhi M, Bakhsheshi-Rad HR, Jahanshahi M, Hasanpour S, Pagan E, Dolatshahi-Pirouz A, Zhang YS, Kundu SC, Akbari M. Hyaluronic acid (HA)-based silk fibroin/zinc oxide core-shell electrospun dressing for burn wound management. Macromol Biosci, 2020; 2020:1900328.

Halib N, Perrone F, Cemazar M, Dapas B, Farra R, Abrami M, Chiarappa G, Forte G, Zanconati F, Pozzato G. Potential applications of nanocellulose-containing materials in the biomedical field. Materials, 2017; 10(8):1-31.

Hanif Z, Ahmed FR, Shin SW, Kim Y-K, Um SH. Size-and dosedependent toxicity of cellulose nanocrystals $(\mathrm{CNC})$ on human fibroblasts and colon adenocarcinoma. Colloids Surf B Biointerfaces, 2014; 119(2014):162-5.

Hu D, Cui Y, Mo K, Wang J, Huang Y, Miao X, Lin J, Chang C. Ultrahigh strength nanocomposite hydrogels designed by locking oriented tunicate cellulose nanocrystals in polymeric networks. Compos B Eng, 2020; 197(2020):1-7.

Hussein Y, El-Fakharany EM, Kamoun EA, Loutfy SA, Amin R, Taha TH, Salim S, Amer M. Electrospun PVA/hyaluronic acid/L-arginine nanofibers for wound healing applications: nanofibers optimization and in vitro bioevaluation. Int J Biol Macromol, 2020; 164(1):667-76.

Khattab TA, Fouda MM, Rehan M, Okla MK, Alamri SA, Alaraidh IA, AL-ghamdi AA, Soufan WH, Abdelsalam EM, Allam AA. Novel halochromic cellulose nanowhiskers from rice straw: visual detection of urea. Carbohydr Polym, 2020; 231:1-33.

Kumar A, Han SS. PVA-based hydrogels for tissue engineering: a review. Int J Polym Mater_Polym Biomater, 2017; 66(4):159-82.

Li Q, Renneckar S. Supramolecular structure characterization of molecularly thin cellulose I nanoparticles. Biomacromolecules, 2011; 12(3):650-9.

Li X, Sun Q, Li Q, Kawazoe N, Chen G. Functional hydrogels with tunable structures and properties for tissue engineering applications. Front Chem, 2018; 6(499):1-36. 
Lu P, Hsieh Y-L. Preparation and characterization of cellulose nanocrystals from rice straw. Carbohydr Polym, 2012; 87(1):564-73.

$\mathrm{Lu}$ P, Hsieh Y-L. Preparation and properties of cellulose nanocrystals: rods, spheres, and network. Carbohydr Polym, 2010; 82(2):329-36.

Menas AL, Yanamala N, Farcas MT, Russo M, Friend S, Fournier PM, Star A, Iavicoli I, Shurin GV, Vogel UB. Fibrillar vs crystalline nanocellulose pulmonary epithelial cell responses: cytotoxicity or inflammation? Chemosphere, 2017; 171(2017):671-80.

Mosmann T. Rapid colorimetric assay for cellular growth and survival: application to proliferation and cytotoxicity assays. J Immunol Methods, 1983; 65(1-2):55-63.

Mu R, Hong X, Ni Y, Li Y, Pang J, Wang Q, Xiao J, Zheng Y. Recent trends and applications of cellulose nanocrystals in food industry. Trends Food Sci Technol, 2019; 93(2019):136-144.

Pelegrini BL, Ré F, de Oliveira MM, Fernandes T, de Oliveira JH, Oliveira Junior AG, Girotto EM, Nakamura C, Sampaio AR, Valim A. Cellulose nanocrystals as a sustainable raw material: cytotoxicity and applications on healthcare technology. Macromol Mater Eng, 2019; 304(8):1-32.

Perumal AB, Sellamuthu PS, Nambiar RB, Sadiku ER. Development of polyvinyl alcohol/chitosan bio-nanocomposite films reinforced with cellulose nanocrystals isolated from rice straw. Appl Surf Sci, 2018; 449(2018):591-602.

Rahman M, Islam M, Islam M, Zaman A, Ahmed T, Biswas S, Sharmeen S, Rashid T, Rahman M. Morphological characterization of hydrogels. In Polymers and polymeric composites: a reference series, Springer, Cham. pp 819-63, 2019.

Raut PW, Shitole AA, Khandwekar A, Sharma N. Engineering biomimetic polyurethane using polyethylene glycol and gelatin for bloodcontacting applications. J Mater Sci, 2019; 54(14):10457-72.

Segal L, Creely J, Martin Jr A, Conrad C. An empirical method for estimating the degree of crystallinity of native cellulose using the X-ray diffractometer. Text Res J, 1959; 29(10):786-94.

Sharma A, Thakur M, Bhattacharya M, Mandal T, Goswami S. Commercial application of cellulose nano-composites-a review. Biotechnol Rep, 2019; 21:e00316.

Singla R, Soni S, Kulurkar PM, Kumari A, Mahesh S, Patial V, Padwad YS, Yadav SK. In situ functionalized nanobiocomposites dressings of bamboo cellulose nanocrystals and silver nanoparticles for accelerated wound healing. Carbohydr Polym, 2017a; 155(2017):152-62.
Singla R, Soni S, Patial V, Kulurkar PM, Kumari A, Mahesh S, Padwad YS, Yadav SK. Cytocompatible anti-microbial dressings of syzygium cumini cellulose nanocrystals decorated with silver nanoparticles accelerate acute and diabetic wound healing. Sci Rep, 2017b; 7(1):1-13.

Thakur M, Sharma A, Ahlawat V, Bhattacharya M, Goswami S. Process optimization for the production of cellulose nanocrystals from rice straw derived $\alpha$-cellulose. Mater Sci Energy Technol, 2020; 3(2020):328-34

Tyagi P, Mathew R, Opperman C, Jameel H, Gonzalez R, Lucia L, Hubbe M, Pal L. High-strength antibacterial chitosan-cellulose nanocrystal composite tissue paper. Langmuir, 2018; 35(1):104-12.

Uranga J, Nguyen BT, Si TT, Guerrero P, de la Caba K. The effect of cross-linking with citric acid on the properties of Agar/fish gelatin films. Polym, 2020; 12(2):1-12.

Xu J, Bai H, Wang M, Xia W, Liu X. Properties of hyaluronan/ PVA-SbQ composite films processed by casting. Polym Compos, 2013; 21(1):55-60.

Xu K, Liu C, Kang K, Zheng Z, Wang S, Tang Z, Yang W. Isolation of nanocrystalline cellulose from rice straw and preparation of its biocomposites with chitosan: physicochemical characterization and evaluation of interfacial compatibility. Compos Sci Technol, 2018; 154(2018):8-17.

Xue Y, Chen H, Xu C, Yu D, Xu H, Hu Y. Synthesis of hyaluronic acid hydrogels by crosslinking the mixture of high-molecularweight hyaluronic acid and low-molecular-weight hyaluronic acid with 1 , 4-butanediol diglycidyl ether. RSC Adv, 2020; 10(12):7206-13.

Yin F, Lin L, Zhan S. Preparation and properties of cellulose nanocrystals, gelatin, hyaluronic acid composite hydrogel as wound dressing. J Biomater Sci Polym Ed, 2019; 30(3):190-201.

\section{How to cite this article:}

Hussein Y, Kamoun EA, Loutfy SA, Amen R, Taha TH, Mansour AS, Abdel-Salam AI, Amer M. Plant nanocellulose and its composite hydrogel membranes-based polyvinyl alcohol/hyaluronic acid for biomedical applications: Extraction, characterization, and in vitro bioevaluation. J Appl Pharm Sci, 2021; 11(01):049-060. 